\title{
MÉTODOS DE ABATE E QUALIDADE DA CARNE DE PEIXE
}

\author{
SLAUGHTER METHODS AND FISH MEAT QUALITY
}

\author{
Viegas, E.M.M. ${ }^{1 *}$, Pimenta, F.A. ${ }^{1}$, Previero, T.C. ${ }^{1}$, Gonçalves, L.U. ${ }^{1}$, Durães, J.P. ${ }^{1}$, \\ Ribeiro, M.A.R. ${ }^{1}$ e Oliveira Filho, P.R.C. ${ }^{1}$
}

${ }^{1}$ Departamento de Zootecnia. Faculdade de Zootecnia e Engenharia de Alimentos. Universidade de São Paulo (FZEA-USP). Pirassununga-SP. Brasil. *emviegas@usp.br

\section{Palavras chave adicionals}

Bem estar animal. Estresse. Atordoamento. Processamento. Alterações post mortem.

\section{RESUMO}

Apesar do abate ser um dos maiores fatores de estresse na produção de animais, não existe neste âmbito, legislação específica para os peixes no Brasil. Os métodos de abate ou atordoamento mais utilizados são a imersão em água e gelo, asfixia no ar, eletricidade, narcose por gases e atordoamento percussivo. No entanto, 0 abate ideal é aquele procedimento que considera o bem estar animal e a qualidade da carne. $O$ estresse perimortem pode levar a uma situação de pânico, medo e fuga fazendo com que os peixes utilizem suas reservas energéticas, diminuindo os teores de glicose e ATP postmorteme conseqüentemente o encurtamento do tempo de rigor mortis, e reduzindo o pH muscular. Essas reações também promovem mudanças indesejáveis nas características sensoriais como cor e textura do pescado, além de facilitar o desenvolvimento de microorganismos deteriorantes, diminuindo a vida de prateleira. De maneira geral, observa-se que os métodos de atordoamento/abate por choque elétrico, percussão craniana e super dosagem de anestésico, quando aplicados de maneira correta, causam pouco sofrimento e melhor qualidade da carne. Os abates de peixes por asfixia no ar ou com gelo, choque térmico, narcose com gases e banho de sal, com exceção de alguns casos, causam muito estresse e piora na qualidade da carne. De acordo com a revisão bibliográfica observa-se que não é possível fazer recomendação de uma única metodologia de abate a ser empregada nas varias espécies de peixes, sendo assim necessário ampliar as pesquisas nesta área de maneira a determinar melhores

\section{AdDitionAL KEYWORDS}

Animal welfare. Stress. Stunning. Processing. Post mortem changes.

técnicas de abate, visando à diminuição do sofrimento e obtendo desta maneira um produto ético de qualidade superior. Este trabalho foi elaborado com o objetivo de revisar os trabalhos mais recentemente publicados sobre o efeito dos diferentes métodos do abate sobre o estresse e a qualidade do pescado.

\section{SUMMARY}

Although the slaughtering is one of the most stressful facts during animal breeding, there is no specific legislation about this procedure in Brazil. The most used slaughter or stunning methods include immersion in ice slurry, air asphyxia, electric shock, gas stunning and percussive blow to the head. However, an ideal method must include welfare animal and meat quality. Perimortem stress may lead to a panic, fear and escape situation compelling fish to deplete their energetic reserves lowering glucose and ATP post mortem values and reducing the muscle $\mathrm{pH}$ leading to a short period of rigor mortis. These reactions also cause undesirable changes in sensorial characteristics such as color and texture, and further bacterial development, thus reducing the shelf life. In general, it is observed that the methods of stunning/ slaughter by electric shock, percussion and anesthetic, when applied correctly, cause little pain and better meat quality. The slaughtering of fish by asphyxia in air or ice, gas narcosis and bath salt, except for some cases, cause much stress and poor meat quality. Accordingly the literature review shows that it is not feasible to make a single 


\section{VIEGAS, PIMENTA, PREVIERO, GONÇALVES, DURÃES, RIBEIRO E OLIVEIRA FILHO}

recommendation for slaughtering methodology to be employed in various fish species, thus there is a need for an increased research in this area in order to determine the best techniques of slaughter reducing suffering, and in this way getting a superior product in a ethical way. This paper has the objective to review and analyze the most recently published papers about the effect of different slaughter methods on stress and fish quality.

\section{INTRODUÇÃO}

Em todas as espécies de animais de sangue quente (mamíferos e aves) é comum propiciar bem estar tanto na criação como no abate (van de Vis et al., 2003; Andrade et al., 2009; Santana et al., 2009), e portanto foi estabelecido que a inconsciência e a insensibilização devem ser induzidas de forma rápida sem descuidar do bem estar animal e da qualidade da carne (Lambooij et al., 2002; Terlouw et al., 2008). Contudo, em contraste com os demais animais de criação zootécnica, não existem leis específicas para a proteção dos peixes durante o processo de abate, embora várias pesquisas venham sendo conduzidas no mundo todo.

Com base em estudos anatômicos, fisiológicos e comportamentais, observou-se que os peixes são muito similares às aves $\mathrm{e}$ mamíferos, o que pode evidenciar também a capacidade dos peixes em sentir dor e medo (Lambooij et al., 2002; Lambooij et al., 2006b). Tradicionalmente a escolha dos métodos de abate em peixes é realizada pela sua facilidade de aplicação e custo reduzido. Os métodos tradicionais de abate tais como asfixia (em ar ou no gelo), evisceração, choque térmico não são considerados humanitários, porque provoca sofrimentos desnecessários, estresse, dor e diminuem a qualidade do produto durante o armazenamento (Lambooij et al., 2006b; Wills et al., 2006; Wilkinson et al., 2008). Adicionalmente, o bem estar dos peixes durante toda a fase de criação e não somente no momento do pré-abate e abate é um tema que atualmente tem sido discutido, princi- palmente em países europeus como a Holanda (Morzel e van de Vis, 2003; Lambooij et al., 2006b), Alemanha e o Reino Unido (Robb e Roth, 2003). Esta revisão concentra-se na citação e análise de artigos científicos mais recentemente publicados referentes a influência dos métodos de atordoamento e morte de peixes cultivados sobre parâmetros de bem estar e qualidade da carne.

\section{BEM ESTAR ANIMAL E MÉTODOS DE ABATE}

O abate dos peixes é um processo que pode ser realizado em um ou dois estágios. No primeiro, os animais são atordoados e insensibilizados, e no segundo, ocorre o sacrifício, que pode ser realizado por diversos métodos. Essas etapas podem acontecer simultaneamente ou em operações distintas, porém o tempo do atordoamento até a morte do animal deve ser minimizado para evitar a recuperação da consciência antes que ocorra a morte (Lines et al., 2003; Jittinandana et al., 2005).

O choque térmico com água e gelo é um dos métodos de abate mais utilizados nas principais espécies de peixes de cultivo marinho e continental, tais como: bagre africano (Clarias gariepinus) (Lambooij et al., 2006a), carpa capim (Ctenopharyngodon idella) (Scherer et al., 2006), sea bream (Sparus aurata) (Huidobro et al., 2001; Tejada e Huidobro, 2002; Bagni et al., 2007; Alvarez et al., 2008), sea bass (Dicentrarchus labrax), (Bagni et al., 2007; Knowles et al., 2007; Acerete et al., 2009), salmão (Salmo salar), (Erikson et al., 2006), turbot (Psetta máxima), (Morzel et al.,2002; Knowles et al., 2008) e truta arco-íris (Oncorhynchus mykiss) (Ozogul e Ozogul, 2004). No entanto, a eficiência desta técnica sobre o bem estar animal tem sido contestada principalmente por causar morte lenta. Por exemplo, para a truta arco-íris (Oncorhynchus mykiss) o tempo para a insensibilização varia de 28 a 198 min, para 


\section{MÉTODOS DE ABATE E QUALIDADE DA CARNE DE PEIXE}

o salmão (Salmo salar) é de aproximadamente 60 min, para o turbot (Psetta máxima) é de $20 \mathrm{~min}$, para o sea bream (Sparus aurata) 20-40 min, para o sea bass (Dicentrarchus labrax) é de 20 min e para a enguia (Anguilla anguilla) $12 \mathrm{~min}$ (Poli et al., 2005). Lambooij et al. (2006a) observaram que a adição de água e gelo, com a temperatura passando de $24^{\circ} \mathrm{C}$ para $0,1^{\circ} \mathrm{C}$ nos tanques de cultivo de bagre africano (Clarias gariepinus) causou movimentos de fuga e aumento nos batimentos cardíacos antes da insensibilização, sendo, portanto, um método estressor para esta espécie de peixe. Para o salmão do Atlântico (Salmo salar) o resfriamento de peixes vivos com e sem a adição de $\mathrm{CO}_{2}$ na água mostraram-se como métodos ineficazes na insensibilização e na perda de consciência (Roth et al., 2006). Em climas quentes, quando os peixes são imersos em água e gelo, o choque térmico pode causar imobilidade e aparente insensibilidade, mas é possível que os animais embora paralisados possam estar ainda vivos (Lines et al., 2003; Lines e Spence, 2011).

O abate por imersão em água saturada com $\mathrm{CO}_{2}$ tem sido estudado principalmente em salmões (Salmo salar) cultivados (Roth et al., 2002; Roth et al., 2005; Roth et al., 2006; Erikson et al., 2006; Lines e Spence, 2011), truta arco-íris (Oncorhynchus mykiss) (Marx et al., 1997; Roth et al., 2005), sea bass (Dicentrarchus labrax) (Acerete et al., 2009), carpa comum (Cyprinus carpio) (Marx et al., 1997), enguia (Anguilla anguilla) (Marx et al., 1997). Essa técnica apesar de muito utilizada por manter a qualidade da carne após o abate, é considerada estressante pelo fato do peixe sofrer com acidificação da água causada pela saturação de $\mathrm{CO}_{2}$, que se dissolve originando $\mathrm{H}_{2} \mathrm{CO}_{3}$ em equilíbrio com $\mathrm{HCO}_{3}$ e $\mathrm{H}^{+}$ indicado por uma rápida e violenta reação de tentativa de escape (Poli et al., 2005; Lines e Spence, 2011; Erikson et al., 2011). Alguns trabalhos mostram que a resposta do salmão do Atlântico (Salmo salar) ao ambiente ácido e hipóxico formado durante o atordoamento por $\mathrm{CO}_{2}$ provoca estresse agudo e tentativa de fuga, causando a entrada precoce no estado de rigor mortis e consequentemente à flacidez muscular precoce (Robb e Roth, 2003; Poli et al., 2005; Roth et al., 2006). O atordoamento por $\mathrm{CO}_{2}$ em Artic char (Salvelinus alpinus) não é efetivo e está associado com o esforço de fuga que leva a redução do $\mathrm{pH}$ muscular durante o abate e após 24 horas de armazenamento (Jittinandanna et al., 2005). Baseados nestas informações, estudos têm sido realizados para se avaliar o potencial de outros gases ou mistura de gases capazes de atordoar os peixes com menor sofrimento. Em truta arco-íris (Oncorhynchus mykiss) os níveis de ATP do músculo dos peixes submetidos à narcose com nitrogênio foram maiores do que nos peixes asfixiados ao ar, demonstrando potencial utilização deste gás como método de atordoamento para esta espécie (Wills et al., 2006). No entanto, salmões do Atlântico (Salmo salar) expostos ao nitrogênio apresentam fortes reações aversivas e dificuldade de sedação, não sendo recomendado como método de atordoamento para esta espécie (Erikson, 2011).

Recentemente o monóxido de carbono começou a ser investigado para o atordoamento de peixes, atuando como agente antioxidante no músculo e observase ter habilidade de inibir a metamioglobina. Em salmões do Atlântico (Salmo salar) expostos ao monóxido de carbono não ocorreram reações adversas facilitando o abate posterior por percussão craniana, embora sua utilização tenha causado diminuição do pH muscular e entrada e saída mais rápida no estado de rigor mortis. Este resultado pode ter ocorrido pela maior afinidade do monóxido de carbono com a proteína heme do que com que o oxigênio, diminuindo o transporte de oxigênio pela hemoglobina e desta forma alterando o metabolismo de aeróbio para anaeróbio (Bjørlykke et al., 2011). 
Em países como a Alemanha e Holanda, o abate tradicional de enguias (Anguilla anguilla) que implicava na remoção do muco corporal através da colocação dos animais em um compartimento com sal marinho até a morte, foi proibido por causar reações de fuga muito violentas, olhos mais opacos e irritações nas mucosas (Morzel e van de Vis, 2003). Atualmente, nestes países o único método permitido para o sacrifício ou atordoamento das enguias é por choque elétrico (Lambooij et al., 2002).

Uma forma de abate muito discutida atualmente é o uso de eletricidade, tanto para atordoamento quanto para abate, uma vez que promove a transição rápida para a insensibilidade e possibilita sua execução individualmente ou em lotes (Lambooij et al., 2002; Lambooij et al., 2006b). Esta técnica tem sido estudada em peixes como o arenque (Clupea harengus) (Nordgreen et al., 2008), carpa comum (Cyprinus carpio), (Lambooij et al., 2007), carpa capim (Ctenopharyngodon idella) (Scherer et al., 2006), sea bass (Dicentrarchus labrax) (Knowles et al., 2007), salmão (Salmo salar) (Roth et al., 2002; Robb e Roth, 2003; Roth et al., 2012), bagre africano (Clarias gariepinus), (Lambooij et al., 2006a), enguia (Anguilla anguilla) (Robb et al., 2002; Morzel e van de Vis, 2003), sea bream (Sparus aurata) (Giuffrida et al., 2007), tilápia do Nilo (Oreochromis niloticus), (Lambooij et al., 2008), turbot (Psetta maxima) (Morzel et al., 2002; Roth et al., 2007; Knowles et al., 2008), truta arco-íris (Oncorhynchus mykiss) (Lines et al., 2003; Lines e Kestin, 2005; Giuffrida et al., 2007). No entanto, para estabelecer a metodologia mais adequada de choque elétrico existe a necessidade de conhecimento da correta voltagem, freqüência, tipo de corrente elétrica e tempo de exposição para cada espécie de peixe (Robb e Roth, 2003; Nordgreen et al., 2008), pois caso se aplique uma corrente elétrica muito forte ou inadequada pode haver quebra de ossos e hemorragias que irão comprometer a qualidade dos filés, com o aparecimento de manchas de sangue (Lines et al., 2003). Bagres africanos (Clarias gariepinus) submetidos ao choque elétrico durante 5 segundos apresentaram perda de consciência sem recuperação, até o abate por decapitação ou imersão em água e gelo (Lambooij et al., 2006a). Em salmões do Atlântico (Salmo salar) a insensibilização pode ser alcançada pelo uso de corrente alternada, pois além da perda imediata de consciência, ocorre a imobilidade, permitindo o abate e processamento de lotes inteiros, diminuindo assim o manuseio (Robb e Roth , 2003). Em outro estudo com salmões do Atlântico, observou-se que o $\mathrm{pH}$ muscular e índice de rigor mortis não apresentaram diferença $(\mathrm{p}<0,05)$ entre $\mathrm{o}$ abate por choque elétrico $(60 \mathrm{~V}, 100 \mathrm{~Hz}$ por 6 s) e percussão craniana, concluindo que a aplicação correta da corrente elétrica produz igualmente um bom resultado quanto a qualidade da carne quando comparado com a percussão craniana (Roth et al., 2012). O método de abate por choque elétrico $(60 \mathrm{~V}$ por $5 \mathrm{~s})$, apresentou maior $(\mathrm{p}<0,05)$ relação ATP/IMP e menores $(p<0,05)$ valores de TBARs que o corte de brânquias ou asfixia no ar em truta arco-íris (Oncorhynchus mykiss), concluindo ser este o método menos estressante para esta espécie (Giuffrida et al., 2007).

\section{MÉTODOS DE ABATE E PARAMETROS DE QUALIDADE DA CARNE}

O fenômeno de rigor mortis consiste na mudança da textura da carne do pescado, passando de macia e flexível (pré-rigor), para rígida e inflexível (rigor completo) e novamente macia, porém não tão flexível (pós-rigor) (Sikorski, 1990). O método mais utilizado para medir o índice de rigor mortis é o desenvolvido por Bito et al. (1983), o qual avalia a diminuição da curvatura inicial do pescado durante o período de pré-rigor até o rigor mortis total. 


\section{MÉTODOS DE ABATE E QUALIDADE DA CARNE DE PEIXE}

Os métodos de abate do pescado que causam estresse conduzem a um rápido consumo nas suas reservas de glicogênio e ATP, produzindo ácido lático e conseqüentemente, diminuindo o $\mathrm{pH}$ da carne (Rahmanifarah et al., 2011). Valores iniciais baixos de $\mathrm{pH}$ post mortem estão associados ao alto estresse ante mortem (Thomas et al., 1999; Skjervold et al., 2001). Assim, os animais que se esforçam antes e durante o abate entram em rigor mortis mais rapidamente (Erikson et al., 1997; Bagni et al., 2007), afetando a qualidade do pescado e diminuindo a vida de prateleira (Bosworth et al., 2007).

Huidobro et al. (2001) verificaram que o gilthead seabream (Sparus aurata) é abatido com maior rapidez (menos de $20 \mathrm{~min}$ ) utilizando-se gelo líquido (água salgada a $2,2^{\circ} \mathrm{C}$ ), proporcionando maior tempo no estado de rigor mortis, quando comparado com a água e gelo (após $40 \mathrm{~min}$ ). O gelo líquido apesar de promover melhores características de conservação, causa a descoloração dos olhos, resultando na diminuição do seu valor comercial.

A utilização de dióxido de carbono $\left(\mathrm{CO}_{2}\right)$ como um atordoante prévio ao abate de bacalhau do Atlântico (Gadus morhua) seguido de percussão craniana demonstrou que os peixes tratados com $\mathrm{CO}_{2}$ entraram e saíram do estado de rigor mortis em menor tempo quando comparados aos abatidos somente com percussão craniana, comprovado pelo menor $(\mathrm{p}<0,05)$ valor de $\mathrm{pH}$ (Kristoffersen et al., 2006). Em outro estudo com bacalhau do Atlântico (Gadus morhua) observou-se que o atordoamento por choque elétrico ( $41 \mathrm{~V}$ e $0,2 \mathrm{~A})$ durante 18 a $27 \mathrm{~s}$, causou maior gasto de energia e conseqüentemente entrada em rigor mortis total mais rapidamente $(\mathrm{p}<0,05)(13$ a $16 \mathrm{~h})$ em relação ao tratamento com anestésico (AQUI-S ${ }^{\mathrm{TM}}$ ) (39 h) (Digre et al., 2010).

Para o salmão do Atlântico (Salmo salar) o abate por narcose com $\mathrm{CO}_{2}$, promoveu a entrada no rigor mortis mais rapidamente (12 horas) do que choque elétrico e percussão craniana (36 horas), provocado pelo pânico devido a hipoxia, causando grande consumo de glicogênio (Roth et al., 2002). O choque elétrico acelerou a entrada no rigor mortis (6 horas) em turbot (Psetta máxima) em relação àqueles submetidos ao choque térmico (24 a 48 horas) e por percussão craniana (96 horas) (Morzel et al., 2002). Na carpa comum (Cyprinus carpio) a asfixia causou entrada mais rápida em rigor mortis total $(3 \mathrm{~h})$ em relação a narcose por $\mathrm{CO}_{2}(12$ h), hipotermia (36 h) e anestésico (óleo de cravo) $(60 \mathrm{~h})$. Nos métodos de atordoamento que causaram rápida entrada em rigor mortis (asfixia, narcose por $\mathrm{CO}_{2}$ e hipotermia) também foi observado comportamento aversivo. Em contrapartida o anestésico causou efeitos menos aversivos, além de melhor qualidade da carne, sendo, portanto um potencial método de atordoamento de carpas (Rahmanifarah et al., 2011).

A textura dos alimentos é um atributo de qualidade que envolve a percepção de características mecânicas (suculência, dureza, elasticidade, etc.), geométricas (tamanho) e de superfície. Entre as características relacionadas à textura, a dureza da carne é de fundamental importância para o consumidor e define o valor comercial do produto (Jain et al., 2007). Considerando as características próprias da textura do pescado, o fenômeno gaping merece destaque, uma vez que é economicamente importante por dificultar o processamento e causar o desagregamento do filé. O gaping é originário na ruptura do tecido conjuntivo que causa a quebra dos blocos de miotomas (Robb et al., 2000).

De maneira geral, a textura da carne de pescado pode ser afetada por procedimentos realizados antes (Bosworth et al., 2007), durante ou após (Roth et al., 2006) o abate. Em salmões do Atlântico (Salmo salar) anestesiados por iso-eugenol (Aqui- $\mathrm{s}^{\circledR}$ ) apresentaram fílés com maior $(\mathrm{p}<0,05)$ força de compressão e resistência ao corte $(38,5$ e $50,6 \mathrm{~N}$, respectivamente) que aqueles atordoados por $\mathrm{CO}_{2}(35,8$ e $48,6 \mathrm{~N}$, respecti- 
vamente) (Kiessling et al., 2004). Filés de salmões atordoados com $\mathrm{CO}_{2}$ apresentam menor força de corte, em comparação àqueles atordoados com choque elétrico ou com percussão craniana, o que pode ser considerado um método não adequado quanto à qualidade da carne (Roth et al., 2002). O mesmo ocorre com enguias (Anguilla anguilla) sacrificadas com gelo líquido as quais apresentam piora da qualidade da carne quando comparadas com choque elétrico (Morzel e van de Vis, 2003).

A capacidade de retenção de água é a habilidade da carne em reter a água contida em sua estrutura. Para o consumidor, essa característica está relacionada com o aspecto antes e durante a cocção e com a palatabilidade do produto final, e é dependente, entre outros fatores, do $\mathrm{pH}$, da força iônica e pressão osmótica (Olivo, 2004). Em pescado submetido a um estresse préabate são observadas modificações indesejáveis como maciez excessiva, o aumento na incidência de gaping e diminuição da capacidade de retenção de água (Roth et al., 2002; Ozogul e Ozogul, 2004; Erikson et al., 2006; Lambooij et al., 2006a).

A cor da carne dos peixes é um dos principais parâmetros avaliados pelos consumidores (Knowles et al., 2008). Em turbot (Psetta máxima) ou carpa capim (Ctenopharyngodon idella) submetidos ao abate por água e gelo ou choque elétrico não foi observado diferença nos parâmetros de luminosidade $\left(\mathrm{L}^{*}\right)$, intensidade da cor vermelha-verde $\left(a^{*}\right)$ e intensidade da cor amarela-azul ( $\left.\mathrm{b}^{*}\right)$ dos filés (Scherer et al., 2005; Knowles et al., 2008). Roth et al. (2009) também não observaram variações na luminosidade $\left(\mathrm{L}^{*}\right)$ dos filés de salmões (Salmo salar) submetidos ao choque elétrico ou percussão craniana. Contrariamente, em carpa comum (Cyprinus carpio) submetida a narcose com $\mathrm{CO}$ foi observado maior valor de $\mathrm{L}^{*}$ e menor de a* nos filés em relação ao tratamento com anestésico, água e gelo e asfixia, respectivamente (Rahmanifarah et al., 2011). O armaze- namento do pescado em gelo pode causar mudanças na coloração do músculo. Em estudo com filés de meagre (Argyrosomus regius), armazenados em gelo durante 18 dias, foi observado aumento nos valores de $\mathrm{L}^{*}, \mathrm{~b}^{*}$ e diminuição da dureza, mostrando as mudanças físicas que ocorrem durante a armazenagem em gelo (Hernandez et al., 2009).

Em geral não tem sido verificadas diferenças nos aspectos sensoriais entre os diferentes métodos de abate para peixes marinhos ou de água doce (Scherer et al., 2005; Knowles et al., 2007; Knowles et al., 2008). No entanto alguns estudos demonstram alterações como olhos saltados e presença de sangue na órbita ocular para a truta arco-íris (Oncorhynchus mykiss) causados pelo abate com pistola pneumática (Özogul e Özogul, 2004) e carne mais avermelhada e firme em enguias (Anguilla anguila) submetidas a narcose com $\mathrm{CO}_{2}$ (Morzel e van de Vis, 2003).

Os métodos químicos mais utilizados para avaliar o frescor do pescado são a quantificação de ATP e seus produtos metabolizáveis. Maiores níveis de ATP foram encontradas em truta arco-íris abatidas por percussão craniana quando comparadas com os peixes insensibilizados por nitrogênio ou asfixia ao ar (Wills et al., 2006). Espécies de peixes de água doce nativas da região Amazônica foram objetos de estudo de Batista et al. (2004) e Almeida et al. (2005), respectivamente com matrinxã (Brycon cephalus) e tambaqui (Colossoma macropomum), abatidos por asfixia em camadas de gelo. Analisando o valor de K para matrinxã, foi observado que houve um aumento de $2 \%$ no primeiro dia para $19 \%$ após 16 dias de armazenagem refrigerada e para o tambaqui encontrou-se valor de K de $12 \%$ em período próximo de armazenamento.

Outro componente químico que serve para avaliar o grau de frescor em pescado são os compostos de bases nitrogenadas voláteis (BNV) que compreendem principalmente em amônia, trimetilamina, dime- 


\section{MÉTODOS DE ABATE E QUALIDADE DA CARNE DE PEIXE}

tilamina, e são originários da OTMA e decomposição de nucleotídeos e à desaminação dos aminoácidos por microorganismos (Contreras-Guzmán, 2002). O abate de tilápias do Nilo em água e gelo ou por asfixia com $\mathrm{CO}_{2}$ não causou $(p>0,05)$ diferenças nos teores de BNV durante o período de armazenagem de até 18 dias, encontrando valores próximos de $19 \mathrm{mg} /$ $100 \mathrm{~g}$ (Albuquerque et al., 2004). Em trutas abatidas por percussão craniana ou asfixia não ocorreram $(\mathrm{p}>0,05)$ variações nas BNV (Duran et al., 2008). Em estudo com carpas capins (Ctenopharyngodon idella) submetidas ao abate por água e gelo ou choque elétrico os valores iniciais de BNV estavam abaixo de $9 \mathrm{mg} / 100 \mathrm{~g}$, não apresentando diferença $(p>0,05)$ entre tratamentos durante 20 dias de armazenagem em gelo (Scherer et al., 2006). Em gilthead seabream (Sparus aurata) abatidas por água e gelo ou gelo líquido também não houve diferença entre os métodos nos valores de TMA, aumentando de 0,4 para $2 \mathrm{mg} / 100 \mathrm{~g}$ após 30 dias de armazenamento em gelo (Tejada e Huidobro, 2002).

\section{CONCLUSÕES}

Um dos maiores desafios da aqüicultura é a produção com qualidade, e para tanto o aperfeiçoamento da cadeia produtiva é essencial. O bem estar animal na produção e principalmente nas fases próximas ao aba-

\section{BIBLIOGRAFIA}

Acerete, L., Reig, L., Alvarez, D., Flos, R. and Tort, L. 2009. Comparison of two stunning/ slaughtering methods on stress response and quality indicators of European sea bass (Dicentrarchus labrax). Aquaculture, 287: 139144.

Albuquerque, W.F., Zapata, J.F.F. and Almeida, R.S. 2004. Estado de frescor, textura e composição muscular da tilápia-do-Nilo (Orechromis niloticus) abatida com dióxido de carbono e armazenada em gelo. Rev Ciên Agron, 35: 264-271. te são cruciais e influenciam de forma intensa na qualidade do produto final. Com o crescimento acentuado e contínuo da aqüicultura no mundo todo, tem-se intensificado as discussões e pesquisas científicas sobre o bem estar dos peixes nas práticas normais de manejo. A correlação amplamente confirmada dos efeitos dos métodos de abate e as condições pré-abate sobre o bem estar, bem como sobre a qualidade de várias espécies de peixes sugerem a necessidade de maiores estudos nesta área. Estes serão cruciais para produzirem dados e recomendações para melhores práticas e futuras legislações. Como as espécies de peixes apresentam diferentes comportamentos fisiológicos em situação de abate ou préabate, a combinação de vários métodos juntos pode ser uma estratégia mais eficiente para manutenção do bem estar e qualidade. A União Européia já possui projetos que prevêem normas específicas para o abate humanitário de pescado. Com essas medidas é provável que muito em breve, para a comercialização, o Brasil e outros países exportadores de pescado devam se adequar, como já é praticado com outras espécies de animais. Portanto, é de fundamental importância o empenho e seriedade de pesquisadores no desenvolvimento de novas tecnologias de métodos de abate mais humanos visando o bem estar e a qualidade do pescado.

Almeida, N.M., Batista, G.M., Kodaira, M., Val, A.L. and Lessi, E. 2005. Determinação do índice de rigor-mortis e sua relação com a degradação dos nucleotídeos em tambaqui (Colossoma macropomum), de piscicultura e conservados em gelo. Ciênc Rural, 35: 698-704.

Alvarez, A., García, B.G., Garrido, M.D. and Hernández, M.D. 2008. The influence of starvation time prior to slaughter on the quality of commercial-sized gilthead seabream (Sparus aurata) during ice storage. Aquaculture, 284: 106-114. 
Andrade, E.N., Silva, R.A.M.S. and Roça, R.O. 2009. Manejo pré-abate de bovinos de corte no pantanal, Brasil. Arch Zootec, 58: 301-304.

Bagni, M., Civitareale, C., Priori, A., Ballerini, A., Finoia, M., Brambill, A. and Marino, G. 2007. Pre-slaughter crowding stress and killing procedures affecting quality and welfare in sea bass (Dicentrarchus labrax) and sea bream (Sparus aurata). Aquaculture, 263: 52-60.

Batista, G.M. Lessi, E., Kodaira, M. and Falcão, P.T. 2004. Alterações bioquímicas post mortem de matrinxã Brycon cephalus (GUNTHER, 1869) procedente da piscicultura, mantido em gelo. Ciênc Tecnol Aliment, 24: 573-581.

Bito, M., Yamanada, K., Mikumo, Y. and Amano, K. 1983. Studies on rigor mortis of fish. I. Difference in the mode of rigor mortis among some varieties of fish by modified Cutting's methods. Bulletin Tokai Reg Fish Res Lab. 109: 89-96.

Bjørlykke, G.A., Roth, B., Sørheimd, O., Kvamme, B.O. and Slinde, E. 2011. The effects of carbon monoxide on Atlantic salmon (Salmo salar L.). Food Chem, 127: 1706-1711.

Bosworth, B.G., Small, B.C., Gregory, D., Kimb, J., Black, S. and Jerrett, A. 2007. Effects of restedharvest using the anesthetic AQUI-S ${ }^{\text {TM }}$ on channel catfish, Ictalurus punctatus, physiology and fillet quality. Aquaculture, 262: 302-318.

Contreras-Guzmán, E.S. 2002. Bioquímica de pescados e invertebrados. CECTA-USACH. Santiago. 309 pp.

Digre, H., Erikson, U., Misimi, E., Lambooij, B. and van de Vis, H., 2010. Electrical stunning of farmed Atlantic cod Gadus morhua L.: a comparison of an industrial and experimental method. Aquacult Res, 41: 1190-1202.

Digre, H., Erikson, U., Skaret, J., Lea, P., GallartJornet, L. and Misimi, E. 2011. Biochemical, physical and sensory quality of ice-stored Atlantic cod (Gadus morhua) as affected by pre-slaughter stress, percussion stunning and AQUI-S ${ }^{\mathrm{TM}}$ anaesthesia. Eur Food Res Technol, 233: 447-456.

Duran, A., Erdemli, U., Karakaya, M. and Yilmaz, M.T. 2008. Effects of slaughter methods on physical, biochemical and microbiological quality of rainbow trout Oncorhynchus mykiss and mirror carp Cyprinus carpio ûlleted in pre-, inor post-rigor periods. Fish Sci, 74: 1146-1156. Erikson, U. 2011. Assessment of different stun- ning methods and recovery of farmed Atlantic salmon (Salmo salar): isoeugenol, nitrogen and three levels of carbon dioxide. Anim Welf, 20: 365-375.

Erikson, U., Sigholt, T. and Seland, A. 1997. Handling stress and water quality during live transportation and slaughter of Atlantic salmon (Salmo salar). Aquaculture, 149: 243-252.

Erikson, U., Hultmann, L. and Steen, J.E. 2006. Live chilling of Atlantic salmon (Salmo salar) combined with mild carbon dioxide anesthesia. I. Establishing a method for large-scale processing of farmed fish. Aquaculture, 252: 183-198.

Erikson, U., Digre, H. and Misimi, E. 2011. Effects of perimortem stress on farmed Atlantic cod product quality: a baseline study. J Food Sci, 76: 251-261.

Giuffrida, A. Pennisi, L., Ziino, G., Fortino, L., Valvo, G., Marino, S. and Panebianco, A. 2007. Influence of slaughtering method on some aspects of quality of gilthead seabream and smoked rainbow trout. Vet Res Commun, 31: 437-446.

Hernández, M.D., López, M.B., Álvarez, A., Ferrandini, E., García, B. and Garrido, M.D. 2009. Sensory, physical, chemical and microbiological changes in aquaculture meager (Argyrosomus regius) fillets during ice storage. Food Chem, 11: 237-245.

Huidobro, A., Mendes, R. and Nunes, M.L. 2001. Slaughtering of gilthead seabream (Sparus aurata) in liquid ice: influence on fish quality. Eur Food Res Technol, 213: 267-272.

Jain, D., Pathare, P.B. and Manikantan, M.R. 2007. Evaluation of texture parameters of Rohu fish (Labeo rohita) during iced storage. J Food Eng, 81: 336-340.

Jittinandana, S., Kenney, P.B., Mazik, P.M., Danley, M., Nelson, C.D., Kiser, R.A. and Hankins, J.A. 2005. Transport and stunning affect quality of artic char fillets. J Muscle Foods, 16: 274-288.

Kiessling, A., Espe, M., Ruohonen, K. and Morkore, T. 2004. Texture, gaping and colour of fish and frozen Atlantic salmon flesh as affected by préslaughter iso-eugenol or $\mathrm{CO}_{2}$ anesthesia. Aquaculture, 236: 645-657.

Knowles, T.G., Brown, S.N., Warriss, P.D., Lines, J., Tinarwo, A., Bravo, A., Carvalho, H. and Gonçalves, A. 2007. Effect of electrical stunning 


\section{MÉTODOS DE ABATE E QUALIDADE DA CARNE DE PEIXE}

at slaughter on the carcass, flesh and eating of farmed sea bass (Dicentrarchus labrax). Aquac Res, 38: 1732-1741.

Knowles, T.G., Brown, S.N., Warriss, P.D., Lines, J., Tinarwo, A. and Sendon, M. 2008. Effect of electrical stunning at slaughter on the quality of farmed turbot (Psetta maxima). Aquac Res, 39: 1731-1738.

Kristoffersen, S., Tobiassen, T., Steinsund, V. and Ragnar L. Olsen. 2006. Slaughter stress, postmortem muscle $\mathrm{pH}$ and rigor development in farmed Atlantic cod (Gadus morhua L.). Int $J$ Food Sci Technol, 41: 130-159.

Lambooij, E., van de Vis, J.W., Kuhlmann, H., Munkner, W., Oehlenschlager, J., Kloosterboer, R.J. and Pieterse, C. 2002. A feasible method for humane slaughter of eel (Anguilla anguilla L.): electrical stunning in fresh water prior to gutting. Aquac Res, 33: 643-652.

Lambooij, E., Kloosterboer, K., Gerritzen, M.A., André, G., Veldman, M. and van de Vis, $H$. 2006a. Electrical stunning followed by decapitation or chilling of African catfish (Clarias gariepinus): assessment of behavioral and neural parameters and product quality. Aquac Res, 37: 61-70.

Lambooij, E., Kloosterboer, K., Gerritzen, M.A. and van de Vis, H. 2006b. Assessment of electrical stunning in fresh water of African Catfish (Clarias gariepinus) and chilling in ice water for loss of consciousness and sensibility. Aquaculture, 254: 388-395.

Lambooij, E., Bialowas, H., van den Boogaart, J.G.M. and van de Vis, J.W. 2007. Electrical and percussive stunning of the common carp ( $\mathrm{Cy}$ prinus carpio L.): Neurological and behavioural assessment. Aquacult Eng, 37: 171-179.

Lambooij, E., Gerritze, M.A., Reimert, H., Burggraaf, D., van de Vis, H. 2008. Humane protocol for electro-stunning and killing of Nile tilapia in fresh water. Aquaculture, 275: 88-95.

Lines, J. and Kestin, S. 2005. Electric stunning of trout: power reduction using a two-stage stun. Aquacult Eng, 32: 483-491.

Lines, J.A. and Spence, J. 2011. Safeguarding the welfare of farmed fish at harvest. Fish Physiol Biochem, doi: 10.1007/s10695-011-9561-5.

Lines, J.A., Robb, D.H.; Kestin, S.C., Crook, S.C. and Benson, T.2003. Electric stunning: a humane slaughter method for trout. Aquacult Eng, 28:
141-154.

Marx, H., Brunner, B., Weinzierl, W., Holfmann, R. and Stolle, A. 1997. Methods of stunning freshwater fish: impact on meat quality and aspects of animal welfare. $Z$ Leesm Uters Forsch, 204: 282-286.

Morzel, M. and van de Vis, H. 2003. Effects of the slaughter method on the quality of raw and smoked eels (Anguilla Anguilla L.). Aquac Res, 34: 1-11.

Morzel, M., Sohier, D. and van de Vis, H. 2002. Evaluation of slaughtering methods for turbot with respect to animal welfare and flesh quality. J Sci Food Agri, 82: 19-28.

Nordgreen, A.H., Slinde, E., Moller, D. and Roth, B. 2008. Effect of various electric field strengths and current durations on stunning and spinal injuries of Atlantic herring. J Aqua Anim Heal, 20: 110-115.

Olivo, R. 2004. Atualidades na qualidade da carne de aves. Rev Nac Carne, 28: 38-50.

Özogul, Y. and Özogul, F. 2004. Effects of slaughtering methods on sensory, chemical and microbiological quality of rainbow trout (Onchorynchus mykiss) stored in ice and MAP. Eur Food Res Technol, 219: 211-216.

Poli, B., Parisi, G., Scappini, F. and Zampacavallo, G. 2005. Fish welfare and quality as affected by pre-slaughter and slaughter management. Aquac Int, 13: 29-49.

Rahmanifarah, K., Shabanpour, B. and Sattari, A. 2011. Effects of clove oil on behavior and flesh quality of common carp (Cyprinus carpio L.) in comparison with pre-slaughter $\mathrm{CO}_{2}$ stunning, chilling and asphyxia. Turk J Fish Aqua Sci, 11: 139-147.

Robb, D.H.F., Kestin, S.C. and Warriss, P.D. 2000. Muscle activity at slaughter: I. Changes in flesh colour and gaping in rainbow trout. Aquaculture, 182: 261-269.

Robb, D.H.F., Wotton, S.B. and van de Vis, J.W. 2002. Preslaughter electrical stunning of eels. Aquac Res, 33: 37-42.

Robb, D.H.F. and Roth, B. 2003. Brain activity of Atlantic salmon (Salmo salar) following electrical stunning using various field strengths and pulse durations. Aquaculture, 216: 363-369.

Roth, B., Moeller, D., Veland, J.O., Imsland, A. and Slinde, E. 2002. The effect of stunning methods on rigor mortis and texture properties of Atlantic 


\section{VIEGAS, PIMENTA, PREVIERO, GONÇALVES, DURÃES, RIBEIRO E OLIVEIRA FILHO}

salmon (Salmo Salar). J Food Sci, 67: 14621466.

Roth, B., Torrissen, O.J. and Slinde, E. 2005. The effect of slaughtering procedures on blood spotting in rainbow trout (Oncorhynchus mykiss) and Atlantic salmon (Salmon salar). Aquaculture, 250: 796-803.

Roth, B., Slinde, E. and Robb, D.H.F. 2006. Field evaluation of live chilling with $\mathrm{CO}_{2}$ on stunning Atlantic salmon (Salmo salar) and the subsequent effect on quality. Aquac Res, 37: 799-804.

Roth, B., Imsland, A., Gunnarsson, S., Foss, A. and Schelvis-Smit, R. 2007. Slaughter quality and rigor contraction in farmed turbot (Scophthalmus maximus); a comparison between different stunning methods. Aquaculture, 272: 754-761.

Roth, B., Birkeland, S. and Oyarzun, F. 2009. Stunning, pre slaughter and filleting conditions of Atlantic salmon and subsequent effect on flesh quality on fresh and smoked fillets. Aquaculture, 289: 350-356.

Roth, B., Grimsbø, E., Slinde, E., Foss, A., Stien, L.H. and Nortvedt, R. 2012. Crowding, pumping and stunning of Atlantic salmon, the subsequent effect on $\mathrm{pH}$ and rigor mortis. Aquaculture, 326329: 178-180.

Santana, A.P., Murata, L.S., MacManus, C.P. and Bernal, F.E.M. 2009. Dosagem de cortisol sanguíneo em suínos submetidos ao manejo préabate e insensibilização elétrica. Arch Zootec, 58: 149-152.

Scherer, R., Augusti, P.R., Steffens, C., Bochi, V.C., Hecktheurer, L.H., Lazzari, R., RadunzNeto, J., Pomblum, S.C.G. and Emanuelli, T. 2005. Effect of slaughter method on postmortem changes of grass carp (Ctenopharyngodon idella) stored in ice. J Food Sci, 70: 348-353.

Scherer, R., Augusti, P.R., Bochi, V.C., Steffens, C., Fries, L.L.M., Daniel, A.N., Kubota, E.H.,
Radunz Neto, J. and Emanuelli, T. 2006. Chemical and microbiological quality of grass carp (Ctenopharyngodon idella) slaughtered by different methods. Food Chem, 99: 136-142.

Sikorski, Z. 1990. Tecnologia de los productos del mar: Recursos, composición nutritiva y conservación. Acribia. Zaragoza. 330 pp.

Skjervold, P.O., Fjaera, S.O., Østby, P.B. and Einen, O. 2001. Live-chilling and crowding stress before slaughter of Atlantic salmon, Salmo salar. Aquaculture, 192: 265-280.

Tejada, M. and Huidobro, A. 2002. Quality of farmed gilthead seabream (Sparus aurata) during ice storage related to the slaughter method and gutting. Eur Food Res Technol, 215: 1-7.

Terlouw, E.M.C., Arnould, C., Auperin, B., Berri, C., Bihan-Duval, E.L., Deiss, V., Lefevre, F., Lensink, B.J. and Mounier, L. 2008. Pre-slaughter conditions, animal stress and welfare: current status and possible future research. Animal, 2: 1501-1517.

Thomas, P.M., Pankhurst, N.W. and Bremner, H.A. 1999. The effect of stress and exercise on post-mortem biochemistry of Atlantic salmon and rainbow trout. J Fish Biol, 5: 1177-1196.

Van de Vis, H., Kestin, S., Robb, D., Oehlenschlager, J., Lambooij, B., Munkner, W., Kuhlmann, H., Kloosterboer, K., Tejada, M., Huidobro, A., Ottera, H., Roth, B., Sorensen, N.K., Akse, L., Byrne, H. and Nesvadba, P. 2003. Is humane slaughter of fish possible for industry? Aquac Res, 34: 211-220.

Wilkinson, R.J., Paton, N. and Porter, M.J.R. 2008. The effects of pre-harvest stress and harvest method on the stress response, rigor onset, muscle $\mathrm{pH}$ and drip loss in barramundi (Lates calcarifer). Aquaculture, 282: 26-32.

Wills, C.C., Zampacavalho, G., Poli, B.M., Proctor, M.R.M. and Henehan, G.T.M. 2006. Nitrogen stunning of rainbow trout. Int. J Food Sci Technol, 41: 395-398. 